\title{
Boundedness and Compactness of Hankel Operators on Large Fock Space
}

\author{
Xiaofeng Wang $(\mathbb{D}$ and Zhicheng Zeng \\ School of Mathematics and Information Science and Key Laboratory of Mathematics and Interdisciplinary Sciences of the \\ Guangdong Higher Education Institute, Guangzhou University, Guangzhou 510006, China
}

Correspondence should be addressed to Zhicheng Zeng; zhichengzeng@e.gzhu.edu.cn

Received 17 September 2021; Revised 4 December 2021; Accepted 14 December 2021; Published 12 January 2022

Academic Editor: Paola Rubbioni

Copyright (c) 2022 Xiaofeng Wang and Zhicheng Zeng. This is an open access article distributed under the Creative Commons Attribution License, which permits unrestricted use, distribution, and reproduction in any medium, provided the original work is properly cited.

We introduce the BMO spaces and use them to characterize complex-valued functions $f$ such that the big Hankel operators $H_{f}$ and $H_{\bar{f}}$ are both bounded or compact from a weighted large Fock space $F^{p}(\phi)$ into a weighted Lebesgue space $L^{p}(\phi)$ when $1 \leq$ $p<\infty$.

\section{Introduction}

Let $\mathbb{C}$ denote the set of complex numbers. For a fixed positive integer $n$, let $\mathbb{C}^{n}$ be the $n$-dimensional Euclidean space. Given $z \in \mathbb{C}^{n}$ and $r>0$, let $B(z, r)$ denote the Euclidean ball defined as

$$
B(z, r)=\left\{w \in \mathbb{C}^{n}:|w-z|<r\right\}
$$

Let $\Delta$ be the Laplace operator and $d v$ denote the Lebesgue volume measure on $\mathbb{C}^{n}$. Suppose $\phi: \mathbb{C}^{n} \longrightarrow \mathbb{R}$ is a $C^{2}$-plurisubharmonic function. We say that $\phi$ belongs to the weight class $\mathscr{W}$, if $\phi$ satisfies the following statements:

(A) There exists $c>0$ such that for $z \in \mathbb{C}^{n}$

$$
\inf _{z \in \mathbb{C}^{n}} \sup _{w \in B(z, c)} \Delta \phi(w)>0
$$

(B) $\Delta \phi$ satisfies the reverse-Hölder's inequality

$$
\|\Delta \phi\|_{L^{\infty}(B(z, r))} \leq C r^{-2 n} \int_{B(z, r)} \Delta \phi d v, \quad \forall z \in \mathbb{C}^{n}, r>0
$$

for some $0<C<\infty$
(C) The eigenvalues of $H_{\phi}$ are comparable; i.e., there exists $\delta_{0}>0$ such that

$$
\left(H_{\phi}(z) u, u\right) \geq \delta_{0} \Delta \phi(z)|u|^{2}, \quad \forall z, u \in \mathbb{C}^{n}
$$

where

$$
H_{\phi}=\left(\frac{\partial^{2} \phi}{\partial z_{j} \partial \bar{z}_{k}}\right)_{j, k}
$$

Let $\phi \in \mathscr{W}$. For $0<p<\infty$, the weighted Lebesgue space $L^{p}(\phi)$ is the set of those measurable functions $f$ on $\mathbb{C}^{n}$ such that

$$
\|f\|_{L^{p}(\phi)}:=\left(\int_{\mathbb{C}^{n}}|f(z)|^{p} e^{-p \phi(z)} d v(z)\right)^{1 / p}<\infty .
$$

For $p=\infty$, the space $L^{\infty}(\phi)$ consists of all measurable functions $f$ on $\mathbb{C}^{n}$ such that

$$
\|f\|_{L^{\infty}(\phi)}:=\sup _{z \in \mathbb{C}^{n}}|f(z)| e^{-\phi(z)}<\infty .
$$


The weighted Fock space $F^{P}(\phi)$ consists of all analytic functions on $\mathbb{C}^{n}$ such that $\|f\|_{L^{p}(\phi)}<\infty$.

The weight class $\mathscr{W}$ is first introduced by Dall'Ara in [1] where the pointwise estimation of the Bergman kernel is given. Recently, the research on the weighted Fock space has been gradually improved. Lv [2] obtained characterizations on the boundedness of the Bergman projection. Arroussi and Tong [3] gave the $L^{p}(\phi)$-norm estimation of the Bergman kernel and studied the boundedness, compactness, and Schatten class membership of the weighted composition operators. Liu and Wang [4] consider the duality of large Fock spaces and obtained equivalent conditions for compactness of weakly localized operators. The weight class $\mathscr{W}$ contains abundant functions. For example, when $\phi(z)$ is replaced by $|z|^{2} / 2, F^{2}(\phi)$ is the classical Fock space (see [5]). The weight function $\phi$ satisfying $d d^{c} \phi \simeq d d^{c}|z|^{2}$ in $[6,7]$ is the member of $\mathscr{W}$. It is interesting that the weight class $\mathscr{W}$ also contains some nonradial weights. An example of nonradial weight is given in [3].

For $1 \leq p<\infty, F^{p}(\phi)$ is a Banach space. In particular, $F^{2}(\phi)$ is a Hilbert space. Let $K_{\phi}(w, z)$ be the Bergman kernel of $F^{2}(\phi)$ and denote $K_{z}(w)=K_{\phi}(w, z)$ for simplicity. We know that $K_{\phi}(\bar{w}, z)=K_{\phi}(z, w)$. The Bergman projection $P$ can be represented as

$$
P g(z)=\int_{\mathbb{C}^{n}} K_{\phi}(z, w) g(w) e^{-2 \phi(w)} d v(w), \quad z \in \mathbb{C}^{n}
$$

Moreover, $P$ is bounded from $L^{p}(\phi)$ to $F^{P}(\phi)$ and $P g=g$ for any $g \in F^{p}(\phi)$ when $1 \leq p<\infty$. See [2, 4] for more details. From [4], we know that

$$
\Gamma=\left\{\sum_{j=1}^{N} a_{j} K_{\phi}\left(\cdot, z_{j}\right): N \in \mathbb{N}, a_{j} \in \mathbb{C}, z_{j} \in \mathbb{C}^{n}, \forall 1 \leq j \leq N\right\},
$$

is dense in $F^{P}(\phi)$. To let $H_{f}$ make sense on $\Gamma$, we naturally consider those measurable functions $f$ in the symbol class $\delta$ defined as

$$
\mathcal{S}=\left\{f: f g \in L^{p}(\phi), \forall g \in \Gamma\right\}
$$

It is obvious that the space $L^{\infty}\left(\mathbb{C}^{n}\right)$ of essentially bounded measurable functions on $\mathbb{C}^{n}$ is contained in $\mathcal{S}$. Given some symbol function $f \in \mathcal{S}$, one defines the big Hankel operator as

$$
H_{f} g=f g-P(f g)
$$
of

Using the property of reproducing kernel, it has the form

$$
H_{f} \mathcal{g}(z)=\int_{\mathbb{C}^{n}}(f(z)-f(w)) g(w) K_{\phi}(z, w) e^{-2 \phi(w)} d v(w), \quad z \in \mathbb{C}^{n}
$$

Hankel operators on Fock spaces and Bergman spaces have been studied by many mathematicians during the past few decades; see $[5,8]$ for instance. The main problem we consider in this paper is to characterize the simultaneous boundedness and compactness of Hankel operators $H_{f}$ and $H_{\bar{f}}$ from $F^{p}(\phi)$ to $L^{p}(\phi)$ with $1 \leq p<\infty$ in terms of suitable defined BMO spaces and VMO spaces. There are exclusive references for this issue both in the setting of Bergman spaces and Fock spaces. See [9-12] for Hankel operators that are simultaneously bounded or compact between the same exponents and [13-16] between different exponents.

This paper is organized as follows. In Section 2, we will give some basic useful results including the $L^{p}(\phi)$-norm as well as pointwise estimation of the reproducing kernel. The space of $\mathrm{BMO}_{r}^{p}$ will be established in Section 3. The decomposition theorem of $\mathrm{BMO}_{r}^{p}$ is given. We will use the $\mathrm{BMO}_{r}^{p}$ space to characterize simultaneously bounded Hankel operators $H_{f}$ and $H_{\bar{f}}$ in Section 4. The space $\mathrm{VMO}_{r}^{p}$ is introduced in Section 5, and it has similar results of the space $\mathrm{BMO}_{r}^{p}$. Compact Hankel operators are characterized in Section 6.

Throughout this paper, we will use $C$ to denote a positive constant whose value may change from line to line, but does not depend on functions being considered. We denote $A \lesssim B$ if there is a positive constant $C$ such that $A \leq C B . A \simeq B$ stands for $A \lesssim B$ and $B \lesssim A$.

\section{Preliminaries}

In this section, we are going to give some basic definitions and results, which will be frequently used. For $z \in \mathbb{C}^{n}$, set

$$
\rho(z):=\sup \left\{r>0: \sup _{w \in B(z, r)} \Delta \phi(w) \leq r^{-2}\right\}
$$

Given $r>0$, write $B^{r}(z)=B(z, r \rho(z))$. We will call $\rho(z)$ a radius function.

Lemma 1. Let $\phi \in \mathscr{W}$. Then, the function $\rho$ satisfies the following properties:

(A) $\rho$ is bounded on $\mathbb{C}^{n}$, that is

$$
\rho(z) \leq \sup _{z \in \mathbb{C}^{n}} \rho(z)=: M, \quad \forall z \in \mathbb{C}^{n}
$$

(B) For $0<r<1$, and $w \in B^{r}(z)$, there holds

$$
(1-r) \rho(z) \leq \rho(w) \leq(1+r) \rho(z) .
$$

Proof. See [3]. 
Let $k_{p, z}$ be the normalized reproducing kernel in $F^{p}(\phi)$, that is

$$
k_{p, z}(w)=\frac{K_{z}(w)}{\left\|K_{z}\right\|_{L^{p}(\phi)}} .
$$

We are going to give some estimations of the reproducing kernel. One can find the proof in [3].

Lemma 2. Let $\phi \in \mathscr{W}$, then one has

(A) For $0<p \leq \infty$

$$
\left\|K_{z}\right\|_{L^{p}(\phi)} \simeq e^{\phi(z)} \rho(z)^{2 n(1-p) / p}, \quad z \in \mathbb{C}^{n} .
$$

(B) There is some $\alpha \in(0,1]$ such that

$$
\left|k_{p, z}(w)\right|^{p} e^{-p \phi(w)} \simeq \rho(z)^{-2 n}, \quad w \in B^{\alpha}(z) .
$$

(C) The set $\left\{k_{p, z}: z \in \mathbb{C}^{n}\right\}$ is bounded in $F^{p}(\phi)$ and $k_{p, z}$ $\longrightarrow 0$ uniformly on every compact subsets of $\mathbb{C}^{n}$ when $|z| \longrightarrow \infty$.

In the following argument, we will always assume $\alpha$ to be a constant as in (B) of Lemma 2. Notice that $\alpha \in(0,1]$, so inequality (15) always holds when $0<r<\alpha$. Before giving the pointwise estimation of the Bergman kernel, we need to introduce a distance function associated with the radius function $\rho$. In fact, we are interested with the Riemannian distance. To describe it explicitly, given $z, w \in \mathbb{C}^{n}$, we define

$$
d(z, w)=\inf _{\gamma} \int_{0}^{1} \frac{\left|\gamma^{\prime}(t)\right|}{\rho(\gamma(t))} d t
$$

where the infimum is taken as $\gamma:[0,1] \longrightarrow \mathbb{C}^{n}$ which varies over the collection of piecewise $C^{1}$-curves with $\gamma(0)=z$ and $\gamma(1)=w$. We define the ball of $d$-metric as

$$
B_{\rho}(z, r)=\left\{w \in \mathbb{C}^{n}: d(z, w)<r\right\} .
$$

The following lemma gives the pointwise estimation of $K_{\phi}(z, w)$.

Lemma 3. There is a constant $\sigma>0$ such that

$$
\left|K_{\phi}(z, w)\right| \lesssim e^{\phi(w)+\phi(z)} \frac{\exp \{-\sigma d(z, w)\}}{\rho(z)^{n} \rho(w)^{n}}, \quad \forall z \in \mathbb{C}^{n} .
$$

Proof. See Theorem 20 of [1].

In what follows, we will always assume $\sigma$ to be a constant as in Lemma 3.

\section{The Space BMO}

For $1 \leq p<\infty$, let $L_{l o c}^{p}\left(\mathbb{C}^{n}\right)$ be the set of all $p$-th locally Lebesgue integrable functions on $\mathbb{C}^{n}$. Suppose $f \in L_{l o c}^{1}\left(\mathbb{C}^{n}\right)$ and $r$ $>0$, the averaging function $\widehat{f}_{r}$ is defined as

$$
\widehat{f}_{r}(z)=\frac{1}{\left|B^{r}(z)\right|} \int_{B^{r}(z)} f(w) d v(w),
$$

where $\left|B^{r}(z)\right|$ is the volume of $B^{r}(z)$. For $f \in L_{l o c}^{p}\left(\mathbb{C}^{n}\right)$, the $p$ -th mean oscillation of $f$ at $z$ is given by

$$
M O_{r}^{p}(f)(z)=\left(\frac{1}{\left|B^{r}(z)\right|} \int_{B^{r}(z)}\left|f(w)-\widehat{f}_{r}(z)\right|^{p} d v(w)\right)^{1 / p} .
$$

Suppose $r>0$ and $1 \leq p<\infty$, the space $\mathrm{BMO}_{r}^{p}$ consists of those functions $f \in L_{\text {loc }}^{p}\left(\mathbb{C}^{n}\right)$ such that

$$
\|f\|_{\mathrm{BMO}_{r}^{p}}=\sup _{z \in \mathbb{C}^{n}} M O_{r}^{p}(f)(z)<\infty .
$$

The following proposition reveals that the function $\widehat{f}_{r}(z)$ in (23) can be replaced by any complex-valued function in $\mathbb{C}^{n}$.

Proposition 4. Let $1 \leq p<\infty, f \in L_{\text {loc }}^{p}\left(\mathbb{C}^{n}\right)$, and $r>0$. Then, $f \in B M O_{r}^{p}$ if and only if there exists a complex-valued function $c(z)$ on $\mathbb{C}^{n}$ such that

$$
\frac{1}{\left|B^{r}(z)\right|} \int_{B^{r}(z)}|f(w)-c(z)|^{p} d v(w) \leq C, \quad z \in \mathbb{C}^{n}
$$

where $C$ is a constant.

Proof. If $f \in \mathrm{BMO}_{r}^{p}$, then (25) holds with $c(z)=\widehat{f}_{r}(z)$ and $C$ $=\|f\|_{\mathrm{BMO}_{r}^{p}}^{p}$.

Conversely, if (25) is satisfied, by the triangle inequality for the $L^{p}$-norm, we obtain

$$
\begin{aligned}
M O_{r}^{p}(f)(z)= & \left(\frac{1}{\left|B^{r}(z)\right|} \int_{B^{r}(z)}\left|f(w)-\widehat{f}_{r}(z)\right|^{p} d v(w)\right)^{1 / p} \\
\leq & \left(\frac{1}{\left|B^{r}(z)\right|} \int_{B^{r}(z)}|f(w)-c(z)|^{p} d v(w)\right)^{1 / p} \\
& +\left(\frac{1}{\left|B^{r}(z)\right|} \int_{B^{r}(z)}\left|\widehat{f}_{r}(z)-c(z)\right|^{p} d v(w)\right)^{1 / p} \\
= & \left(\frac{1}{\left|B^{r}(z)\right|} \int_{B^{r}(z)}|f(w)-c(z)|^{p} d v(w)\right)^{1 / p} \\
& +\left|\widehat{f}_{r}(z)-c(z)\right| .
\end{aligned}
$$


For $1<p<\infty$, by the Hölder's inequality, one can get

$$
\begin{aligned}
\left|\widehat{f}_{r}(z)-c(z)\right| & =\left|\frac{1}{\left|B^{r}(z)\right|} \int_{B^{r}(z)} f(w)-c(z) d v(w)\right| \\
& \leq\left(\frac{1}{\left|B^{r}(z)\right|} \int_{B^{r}(z)}|f(w)-c(z)|^{p} d v(w)\right)^{1 / p} .
\end{aligned}
$$

For $p=1$, it is obvious that

$$
\left|\widehat{f}_{r}(z)-c(z)\right| \leq \frac{1}{\left|B^{r}(z)\right|} \int_{B^{r}(z)}|f(w)-c(z)| d v(w) .
$$

So we have

$$
M O_{r}^{p}(f)(z) \leq 2\left(\frac{1}{\left|B^{r}(z)\right|} \int_{B^{r}(z)}|f(w)-c(z)|^{p} d v(w)\right)^{1 / p}
$$

which implies

$$
\|f\|_{\mathrm{BMO}_{r}^{p}}^{p} \leq 2^{p} C
$$

This completes the proof. $\square$

Let $H\left(B^{r}(z)\right)$ be the set of all analytic functions on $B^{r}(z)$.

Lemma 5. Let $1 \leq p<\infty, f \in L_{l o c}^{p}\left(\mathbb{C}^{n}\right)$, and $r>0$. If for any $z \in \mathbb{C}^{n}$, there exist functions $h_{1}, h_{2} \in H\left(B^{r}(z)\right)$ and constants $C_{1}, C_{2}>0$ such that

$$
\begin{aligned}
& \frac{1}{\left|B^{r}(z)\right|} \int_{B^{r}(z)}\left|f(w)-h_{1}(w)\right|^{p} d v(w) \leq C_{1}, \\
& \frac{1}{\left|B^{r}(z)\right|} \int_{B^{r}(z)}\left|\bar{f}(w)-h_{2}(w)\right|^{p} d v(w) \leq C_{2},
\end{aligned}
$$

then $f \in B M O_{r}^{p}$.

Proof. The approach is similar to [13]. It is well known that, if $v: B(0,1) \longrightarrow \mathbb{R}$ is pluriharmonic, there exists some pluriharmonic function $u: B(0,1) \longrightarrow \mathbb{R}$ such that $u+i v \in H($ $B(0,1))$. Theorem 1 from [17] tells us there is some constant $C>0$ such that

$$
\|u-u(0)\|_{L^{p}(B(0,1), d v)} \leq C\|v\|_{L^{p}(B(0,1), d v)}
$$

Hence, for any $r>0$, by change of variables, we know that, if $v: B^{r}(z) \longrightarrow \mathbb{R}$ is pluriharmonic, there exists a pluriharmonic function $u$ such that $u+i v \in H\left(B^{r}(z)\right)$ and

$$
\|u-u(z)\|_{L^{p}\left(B^{r}(z), d v\right)} \leq C\|v\|_{L^{p}\left(B^{r}(z), d v\right)} .
$$

For $f \in L_{l o c}^{p}\left(\mathbb{C}^{n}\right)$, set $\|f\|_{p, z}=\left(1 /\left|B^{r}(z)\right| \int_{B^{r}(z)}|f|^{p} d v\right)^{1 / p}$.
By the triangle inequality, we have

$$
\begin{aligned}
\left\|\frac{f+\bar{f}}{2}-\frac{h_{1}+h_{2}}{2}\right\|_{p, z} & \leq\left\|\frac{f-h_{1}}{2}\right\|_{p, z}+\left\|\frac{\bar{f}-h_{2}}{2}\right\|_{p, z} \\
& \leq \frac{1}{2}\left(C_{1}^{1 / p}+C_{2}^{1 / p}\right) .
\end{aligned}
$$

Since $f+\bar{f}$ is real-valued, we get

$$
\left\|\operatorname{Im} \frac{h_{1}+h_{2}}{2}\right\|_{p, z} \leq \frac{1}{2}\left(C_{1}^{1 / p}+C_{2}^{1 / p}\right) .
$$

Notice that $h_{1}+h_{2} \in H\left(B^{r}(z)\right)$, by (33), one has

$$
\begin{gathered}
\left\|\operatorname{Re} \frac{h_{1}+h_{2}}{2}-\operatorname{Re} \frac{h_{1}+h_{2}}{2}(z)\right\|_{p, z} \\
\leq C\left\|\operatorname{Im} \frac{h_{1}+h_{2}}{2}\right\|_{p, z} \leq \frac{C}{2}\left(C_{1}^{1 / p}+C_{2}^{1 / p}\right) .
\end{gathered}
$$

By the triangle inequality, there holds

$$
\begin{aligned}
& \left\|\frac{f+\bar{f}}{2}-\operatorname{Re} \frac{h_{1}+h_{2}}{2}(z)\right\|_{p, z} \\
& \leq\left\|\frac{f+\bar{f}}{2}-\operatorname{Re} \frac{h_{1}+h_{2}}{2}\right\|_{p, z} \\
& \quad+\left\|\operatorname{Re} \frac{h_{1}+h_{2}}{2}-\operatorname{Re} \frac{h_{1}+h_{2}}{2}(z)\right\|_{p, z} \\
& \leq\left\|\frac{f+\bar{f}}{2}-\frac{h_{1}+h_{2}}{2}\right\|_{p, z}+\left\|\operatorname{Re} \frac{h_{1}+h_{2}}{2}-\operatorname{Re} \frac{h_{1}+h_{2}}{2}(z)\right\|_{p, z} \\
& \leq \frac{1+C}{2}\left(C_{1}^{1 / p}+C_{2}^{1 / p}\right) .
\end{aligned}
$$

Similarly, there holds

$$
\left\|\operatorname{Im} \frac{f-\bar{f}}{2}-\operatorname{Im} \frac{h_{1}-h_{2}}{2}(z)\right\|_{p, z} \lesssim C_{1}^{1 / p}+C_{2}^{1 / p} .
$$

Set $\quad c(z)=\operatorname{Re}\left(\left(h_{1}+h_{2}\right) / 2\right)(z)+i \cdot \operatorname{Im}\left(\left(h_{1}-h_{2}\right) / 2\right)(z)$. By the triangle inequality again, we have

$$
\|f-c(z)\|_{p, z} \lesssim C_{1}^{1 / p}+C_{2}^{1 / p}
$$

From this and Proposition 4, the desired result follows immediately. $\square$

To arrive at the decomposition theorem, we need to introduce two subspaces of $\mathrm{BMO}_{r}^{p}$ : one is $B O_{r}$, the other is $B A_{r}^{p}$.

For a continuous function $f$ on $\mathbb{C}^{n}$ and radius $r>0$, let

$$
\omega_{r}(f)(z)=\sup \left\{|f(z)-f(w)|: w \in B^{r}(z)\right\} .
$$


The function $\omega_{r}(f)(z)$ is called the oscillation of $f$ at $z$. For $r>0$, let $B O_{r}$ denote the space of all continuous functions $f$ on $\mathbb{C}^{n}$ such that

$$
\|f\|_{B O_{r}}=\sup _{z \in \mathbb{C}^{n}} \omega_{r}(f)(z)<\infty .
$$

Before we give a characterization of functions $f \in B O_{r}$, we need the following lemma.

Lemma 6. There holds

$$
B_{\rho}\left(z, \frac{r}{1+r}\right) \subseteq B^{r}(z) \subseteq B_{\rho}\left(z, \frac{r}{1-r}\right),
$$

for $z \in \mathbb{C}^{n}$ and $r \in(0,1)$.

Proof. If $w \in B^{r}(z)$, take the curve $\gamma(t)=z+t(z-w)$. For $t$ $\in[0,1], \gamma(t) \in B^{r}(z)$. By (15), there holds

$$
\rho(\gamma(t)) \geq(1-r) \rho(z), \quad \forall t \in[0,1] .
$$

It follows that

$$
d(z, w) \leq \int_{0}^{1} \frac{|z-w|}{\rho(\gamma(t))} d t \leq \frac{|z-w|}{(1-r) \rho(z)} \leq \frac{r \rho(z)}{(1-r) \rho(z)}=\frac{r}{1-r},
$$

which makes $B^{r}(z) \subseteq B_{\rho}(z, r /(1-r))$.

If $w \notin B^{r}(z)$, take any piecewise $C^{1}$-curve $\gamma:[0,1] \longrightarrow$ $\mathbb{C}^{n}$ connecting $z$ and $w$, and let $T_{0}$ be the minimum time such that $\left|z-\gamma\left(T_{0}\right)\right|=r \rho(z)$. By (15), there holds

$$
\rho(\gamma(t)) \leq(1+r) \rho(z), \quad \forall t \in\left[0, T_{0}\right) .
$$

This implies that

$$
\int_{0}^{1} \frac{\left|\gamma^{\prime}(t)\right|}{\rho(\gamma(t))} d t \geq \int_{0}^{T_{0}} \frac{\left|\gamma^{\prime}(t)\right|}{\rho(\gamma(t))} d t \geq \int_{0}^{T_{0}} \frac{\left|\gamma^{\prime}(t)\right|}{(1+r) \rho(z)} d t
$$

With the fact that $\int_{0}^{T_{0}}\left|\gamma^{\prime}(t)\right| d t \geq r \rho(z)$, one has $d(z, w$ )$\geq r /(1+r)$. It follows that $B_{\rho}(z, r /(1+r)) \subseteq B^{r}(z)$. The proof is completed.

Proposition 7. Suppose $r \in(0,1)$. Then, the space $B O_{r}$ is independent of $r$. Moreover, a continuous function $f$ on $\mathbb{C}^{n}$ belongs to $\mathrm{BO}_{r}$ if and only if for any $z, w \in \mathbb{C}^{n}$, there exists a constant $C_{f, r}>0$ such that

$$
|f(z)-f(w)| \leq C_{f, r}(d(z, w)+1),
$$

where $C_{f, r} \simeq\|f\|_{B O_{r}}$.

Proof. Suppose $f \in B O_{r}$. Set $M_{r}=r /(1+2 r)<r /(1+r)$. According to Lemma 6 , we get $w \in B^{r}(z)$ and $z \in B^{r}(w)$ whenever $d(z, w) \leq M_{r}$.
If $d(z, w) \leq M_{r}$, then for any $z \in \mathbb{C}^{n}$, there holds

$$
|f(z)-f(w)| \leq \omega_{r}(f)(z) \leq\|f\|_{B O_{r}}(d(z, w)+1) .
$$

Take $C_{f, r}=\|f\|_{B O_{r}}$, then the inequality (47) holds in the case of $d(z, w) \leq M_{r}$.

If $d(z, w)>M_{r}$, we replace points $z_{0}=z, z_{1}, \cdots, z_{N}=w$ on the geodesic in the $d$-metric from $z$ to $w$ in such a way: $d\left(z_{i}, z_{i-1}\right)=M_{r}$ for $i=1,2, \cdots, N-1$ and $d\left(z_{N}, z_{N-1}\right) \leq M_{r}$. Note that it is possible to avoid using the completeness of the $d$-metric. More specifically, we do not have to use geodesics. Instead, we can simply use a curve from $z$ and $w$ whose length in the $d$-metric differs slightly from $d(z, w)$. Since $d$ $(z, w)=(N-1) M_{r}+d\left(z_{N-1}, w\right)$, one can get

$$
M_{r}+d(z, w)=N M_{r}+d\left(z_{N-1}, w\right) \geq N M_{r} .
$$

It follows that

$$
N \leq 1+M_{r}^{-1} d(z, w) \leq\left(2+\frac{1}{r}\right)(d(z, w)+1) .
$$

By the triangle inequality, there holds

$$
|f(z)-f(w)| \leq \sum_{i=1}^{N}\left|f\left(z_{i}\right)-f\left(z_{i-1}\right)\right| \leq \sum_{i=1}^{N} \omega_{r}(f)\left(z_{i}\right) \leq N\|f\|_{B O_{r}} .
$$

Take $C_{f, r}=(2+1 / r)\|f\|_{B O_{r}}$, then the inequality (47) comes from combining (50) and (51).

Conversely, suppose (47) holds. Set $C_{r}=2 r /(1-r)$. According to Lemma 6, we have $d(z, w) \leq C_{r}$ whenever $w$ $\in B^{r}(z)$. By (47), there holds

$$
|f(z)-f(w)| \leq\left(C_{r}+1\right) C_{f, r}, \quad \forall z \in \mathbb{C}^{n}, w \in B^{r}(z) .
$$

It follows that

$$
\|f\|_{B O_{r}} \lesssim C_{f, r} .
$$

This completes the proof. $\square$

From the above proposition, we have proved that $\mathrm{BO}_{r}$ is independent of the radius $r$ for any $r \in(0,1)$. So one can simply write $B O$ for short.

For $1 \leq p<\infty$ and $r>0$, let $B A_{r}^{p}$ denote the space of all locally $p$-th integrable functions $f$ on $\mathbb{C}^{n}$ such that

$$
\|f\|_{B A_{r}^{p}}=\sup _{z \in \mathbb{C}^{n}}\left[\left(\widehat{|f|^{p}}\right)_{r}(z)\right]^{1 / p}<\infty .
$$

Given a Borel measure $\mu$, a radius $r>0$, and $1 \leq t<\infty$, we define the averaging function with respect to $\mu$ as follows:

$$
\widehat{\mu}_{r}(z)=\frac{1}{\left|B^{r}(z)\right|} \int_{B^{r}(z)} d \mu(w), \quad \forall z \in \mathbb{C}^{n} .
$$


Suppose $\mu$ is a Borel measure. Let $r>0$ and $1 \leq t<\infty$; we define the $t$-Berezin transform with respect to $\mu$ as follows:

$$
\tilde{\mu}_{t}(z)=\int_{\mathbb{C}^{n}}\left|k_{t, z}(w)\right|^{t} e^{-t \phi(\mathrm{w})} d \mu(w) .
$$

In particular, for a function $f$ on $\mathbb{C}^{n}$, set $d \mu=f d v$, then

$$
\tilde{f}_{t}(z)=\int_{\mathbb{C}^{n}} f(w)\left|k_{t, z}(w)\right|^{t} e^{-t \phi(w)} d v(w)
$$

For $1 \leq p<\infty$, we call a positive Borel measure $\mu$ on $\mathbb{C}^{n}$ a Carleson measure if there is a positive constant $C$ such that

$$
\left(\int_{\mathbb{C}^{n}}\left|g(w) e^{-\phi(w)}\right|^{p} d \mu(w)\right)^{1 / p} \leq C\|g\|_{L^{p}(\phi)},
$$

for every function $g \in F^{p}(\phi)$. This definition means that the identity mapping

$$
I_{\mu}: F^{p}(\phi) \longrightarrow L^{p}\left(\mathbb{C}^{n}, e^{-p \phi} d \mu\right)
$$

is bounded. The following lemma characterizes the Carleson measure, and one can find it from Theorem 3.2 in [3] and its proof.

Lemma 8. Let $\mu$ be a finite positive Borel measure on $\mathbb{C}^{n}$. Assume $0<p<\infty$. Then, the following conditions are all equivalent:

(A) The measure $\mu$ is a Carleson measure for $F^{p}(\phi)$

(B) The function $\tilde{\mu}_{t} \in L^{\infty}\left(\mathbb{C}^{n}, d v\right)$ for $t>0$

(C) The function $\widehat{\mu}_{r} \in L^{\infty}\left(\mathbb{C}^{n}, d v\right)$ for $0<r<\alpha$. Moreover

$$
\left\|I_{\mu}\right\|^{p} \lesssim\left\|\tilde{\mu}_{t}\right\|_{L^{\infty}\left(\mathbb{C}^{n}, d v\right)} \simeq\left\|\widehat{\mu}_{r}\right\|_{L^{\infty}\left(\mathbb{C}^{n}, d v\right)} .
$$

Set $d \mu=|f|^{p} d \nu$. Then, we have $\widehat{\mu}_{r}(z)=\left(\widehat{|f|^{p}}\right)_{r}(z)$ and $\tilde{\mu}_{t}(z)=\left(\widetilde{|f|^{p}}\right)_{t}(z)$. The following lemma comes immediately.

Lemma 9. Let $1 \leq p<\infty$ and $f \in L_{\text {loc }}^{p}\left(\mathbb{C}^{n}\right)$. Then, the following conditions are all equivalent:

(A) The measure $d \mu=|f|^{p} d v$ is a Carleson measure for $F^{p}(\phi)$

(B) The function $\left(\widetilde{|f|^{p}}\right)_{t} \in L^{\infty}\left(\mathbb{C}^{n}, d v\right)$ for $t>0$

(C) The function $\left(\left.\widehat{|f|}\right|_{r} \in L^{\infty}\left(\mathbb{C}^{n}, d v\right)\right.$ for $0<r<\alpha$, i.e., $f \in B A_{r}^{p}$. Moreover

$$
\left.\left\|I_{\mu}\right\|^{p} \lesssim \| \widetilde{\left(|f|^{p}\right.}\right)_{t}\left\|_{L^{\infty}\left(\mathbb{C}^{n}, d v\right)} \simeq\right\| f \|_{B A_{r}^{p}}^{p}
$$

From the previous lemma, one can see that the space $B$ $A_{r}^{p}$ is independent of the choice of $r \in(0, \alpha)$. So we will write $B A^{p}$ for short.

Lemma 10. Let $p>0, k \in \mathbb{R}$, then there holds

$$
\int_{\mathbb{C}^{n}} \rho(w)^{k} e^{-p d(z, w)} d v(w) \lesssim \rho(z)^{k+2 n} .
$$

Proof. See [2]

Theorem 11. Let $0<r<\alpha, 1 \leq p<\infty$, and $0<t, l<\infty$. Then, the following conditions are all equivalent:

(A) $f \in B M O_{r}^{p}$

(B) $f$ admits a decomposition $f=f_{1}+f_{2}$ where $f_{1} \in B O_{r}$ and $f_{2} \in B A_{r}^{p}$

(C) The function $\int_{\mathbb{C}^{n}}\left|f(w)-\tilde{f}_{t}(z)\right|^{p} \cdot\left|k_{l, z}(w)\right|^{l} e^{-l \phi(w)} d v($ $w)$ is bounded. Moreover

$$
\left\|f_{1}\right\|_{B O_{r}}+\left\|f_{2}\right\|_{B A_{r}^{p}} \simeq\|f\|_{B M O_{r}^{p}} .
$$

Proof. $(\mathrm{A}) \Rightarrow(\mathrm{B})$ : suppose $f \in \mathrm{BMO}_{r}^{p}$. For $w \in B^{r / 4}(z)$, we have $B^{r / 4}(w) \subseteq B^{r}(z)$. In fact, let $\xi \in B^{r / 4}(w)$. Since $0<r<\alpha$ $\leq 1$, using inequality (15), we know that

$$
\left(1-\frac{r}{4}\right) \rho(z) \leq \rho(w) \leq\left(1+\frac{r}{4}\right) \rho(z) .
$$

Applying the triangle inequality, we get

$$
\begin{aligned}
|\xi-z| & \leq|\xi-w|+|w-z|<\frac{r}{4} \rho(w)+\frac{r}{4} \rho(z) \\
& \leq\left(1+\frac{r}{4}\right) \frac{r}{4} \rho(z)+\frac{r}{4} \rho(z)<r \rho(z) .
\end{aligned}
$$

Set $f_{1}=\widehat{f}_{r / 4}$ and $f_{2}=f-f_{1}$. For $1<p<\infty$ and $w \in B^{r / 4}($ $z)$, by the triangle inequality and Hölder's inequality, one can get

$$
\begin{aligned}
\left|f_{1}(z)-f_{1}(w)\right| \leq & \left|f_{1}(z)-\widehat{f}_{r}(z)\right|+\left|\widehat{f}_{r}(z)-f_{1}(w)\right| \\
\leq & \frac{1}{\left|B^{r / 4}(z)\right|} \int_{B^{r / 4}(z)}\left|f(u)-\widehat{f}_{r}(z)\right| d v(u) \\
& +\frac{1}{\left|B^{r / 4}(w)\right|} \int_{B^{r / 4}(w)}\left|f(u)-\widehat{f}_{r}(z)\right| d v(u) \\
\leq & \left(\frac{1}{\left|B^{r}(z)\right|} \int_{B^{r}(z)}\left|f(u)-\widehat{f}_{r}(z)\right|^{p} d v(u)\right)^{1 / p} .
\end{aligned}
$$

For $p=1$ and $w \in B^{r / 4}(z)$, there also holds

$$
\left|f_{1}(z)-f_{1}(w)\right| \lesssim \frac{1}{\left|B^{r}(z)\right|} \int_{B^{r}(z)}\left|f(u)-\widehat{f}_{r}(z)\right| d v(u) .
$$


Thus $f_{1} \in B O_{r / 4}$. By Proposition 7, we have $f_{1} \in B O_{r}$. Moreover,

$$
\left\|f_{1}\right\|_{B O_{r}} \lesssim\|f\|_{\mathrm{BMO}_{r}^{p}}
$$

Next, we prove the part with regard to $f_{2}$. By the triangle inequality, one can get

$$
\begin{aligned}
M O_{r / 4}^{p}(\mathrm{f})(z) \leq & \left(\frac{1}{\left|B^{r / 4}(z)\right|} \int_{B^{r / 4}(z)}\left|f(u)-\widehat{f}_{r}(z)\right|^{p} d v(u)\right)^{1 / p} \\
& +\left|\widehat{f}_{r}(z)-\widehat{f}_{r / 4}(z)\right| .
\end{aligned}
$$

For $1<p<\infty$, using the Hölder's inequality, there holds

$$
\begin{aligned}
\left|\widehat{f}_{r}(z)-\widehat{f}_{r / 4}(z)\right| & =\left|\frac{1}{\left|B^{r / 4}(z)\right|} \int_{B^{r / 4}(z)} f(u)-\widehat{f}_{r}(z) d v(u)\right| \\
& \leq\left(\frac{1}{\left|B^{r / 4}(z)\right|} \int_{B^{r / 4}(z)}\left|f(u)-\widehat{f}_{r}(z)\right|^{p} d v(u)\right)^{1 / p} .
\end{aligned}
$$

For $p=1$, the above inequality is obvious. So we conclude that $M O_{r / 4}^{p}(f)(z) \lesssim M O_{r}^{p}(f)(z)$ for all $z \in \mathbb{C}^{n}$.

$$
\begin{aligned}
\left(\left(\widehat{\left|f_{2}\right|^{p}}\right)_{r / 4}(z)\right)^{1 / p}= & \left(\frac{1}{\left|B^{r / 4}(z)\right|} \int_{B^{r / 4}(z)}\left|f(u)-f_{1}(u)\right|^{p} d v(u)\right)^{1 / p} \\
\leq & \left(\frac{1}{\left|B^{r / 4}(z)\right|} \int_{B^{r / 4}(z)}\left|f(u)-\widehat{f}_{r / 4}(z)\right|^{p} d v(u)\right)^{1 / p} \\
& +\left(\frac{1}{\left|B^{r / 4}(z)\right|} \int_{B^{r / 4}(z)}\left|f_{1}(u)-f_{1}(z)\right|^{p} d v(u)\right)^{1 / p} \\
\leq & M O_{r / 4}^{p}(f)(z)+\omega_{r / 4}\left(f_{1}\right)(z) \\
\leq & M O_{r}^{p}(f)(z)+\omega_{r}\left(f_{1}\right)(z) .
\end{aligned}
$$

Thus, $f_{2} \in B A_{r / 4}^{p}$. By Lemma 9, we have $f_{2} \in B A_{r}^{p}$. Moreover,

$$
\left\|f_{2}\right\|_{B A_{r}^{p}} \lesssim\|f\|_{\mathrm{BMO}_{r}^{p}}
$$

$(\mathrm{B}) \Rightarrow(\mathrm{C})$ : set $f=f_{1}+f_{2}$ where $f_{1} \in B O_{r}$ and $f_{2} \in B A_{r}^{p}$.
By the triangle inequality, there holds

$$
\begin{aligned}
& \left(\int_{\mathbb{C}^{n}}\left|f_{1}(w)-\widetilde{\left(f_{1}\right)_{t}}(z)\right|^{p} \cdot\left|k_{l, z}(w)\right|^{l} e^{-l \phi(w)} d v(w)\right)^{1 / p} \\
& \quad \leq\left(\int_{\mathbb{C}^{n}}\left|f_{1}(w)-f_{1}(z)\right|^{p} \cdot\left|k_{l, z}(w)\right|^{l} e^{-l \phi(w)} d v(w)\right)^{1 / p} \\
& \quad+\left(\int_{\mathbb{C}^{n}}\left|f_{1}(z)-\widetilde{\left(f_{1}\right)_{t}}(z)\right|^{p} \cdot\left|k_{l, z}(w)\right|^{l} e^{-l \phi(w)} d v(w)\right)^{1 / p} \\
& \quad=\left(\int_{\mathbb{C}^{n}}\left|f_{1}(w)-f_{1}(z)\right|^{p} \cdot\left|k_{l, z}(w)\right|^{l} e^{-l \phi(w)} d v(w)\right)^{1 / p} \\
& \quad+\left|f_{1}(z)-\widetilde{\left(f_{1}\right)_{t}}(z)\right| .
\end{aligned}
$$

By (17) and Lemma 3, we have

$$
\left|k_{l, z}(w)\right|^{l} e^{-l \phi(w)} \simeq \rho(z)^{n l-2 n} \rho(w)^{-n l} e^{-\sigma l d(z, w)} .
$$

Since $(x+1)^{a} e^{-b x} \longrightarrow 0$ as $x \longrightarrow+\infty$ for any $a, b>0$, there exists a constant $C$ such that

$$
\sup _{z, w \in \mathbb{C}^{n}}(d(z, w)+1)^{p} e^{-(1 / 2) \sigma l d(z, w)} \leq C .
$$

By Proposition 7, Lemma 10, (74), and (75), one can get

$$
\begin{aligned}
& \int_{\mathbb{C}^{n}}\left|f_{1}(w)-f_{1}(z)\right|^{p} \cdot\left|k_{l, z}(w)\right|^{l} e^{-l \phi(w)} d v(w) \\
& \quad \leq\left\|f_{1}\right\|_{B O_{r}}^{p} \int_{\mathbb{C}^{n}}(d(z, w)+1)^{p} \rho(z)^{n l-2 n} \rho(w)^{-n l} e^{-\sigma l d(z, w)} d v(w) \\
& \quad \leq\left\|f_{1}\right\|_{B O_{r}}^{p} \int_{\mathbb{C}^{n}} \rho(z)^{n l-2 n} \rho(w)^{-n l} e^{-(1 / 2) \sigma l d(z, w)} d v(w) \lesssim\left\|f_{1}\right\|_{B O_{r}}^{p} .
\end{aligned}
$$

For $1<p<\infty$, by Hölder's inequality and (76), we obtain

$$
\begin{aligned}
\left|f_{1}(z)-\widetilde{\left(f_{1}\right)_{t}}(z)\right| & =\left.\left|\int_{\mathbb{C}^{n}}\left(f_{1}(z)-f_{1}(w)\right) \cdot\right| k_{t, z}(w)\right|^{t} e^{-t \phi(w)} d v(w) \mid \\
& \leq\left(\int_{\mathbb{C}^{n}}\left|f_{1}(w)-f_{1}(z)\right|^{p} \cdot\left|k_{t, z}(w)\right|^{t} e^{-t \phi(w)} d v(w)\right)^{1 / p} \\
& \leq\left\|f_{1}\right\|_{B O_{r}} .
\end{aligned}
$$

The same is true for (77) when $p=1$. By (73), (76), and (77), there holds

$$
\left(\int_{\mathbb{C}^{n}}\left|f_{1}(w)-\widetilde{\left(f_{1}\right)_{t}}(z)\right|^{p} \cdot\left|k_{l, z}(w)\right|^{l} e^{-l \phi(w)} d v(w)\right)^{1 / p} \leqslant\left\|f_{1}\right\|_{B O_{r}}
$$

Next, we prove the part with regard to $f_{2}$. For $1<p<\infty$, 
applying Hölder's inequality, we obtain

$$
\widetilde{\mid\left(f_{2}\right)_{t}}(z) \mid \lesssim\left[\left(\widetilde{\left|f_{2}\right|^{p}}\right)_{t}(z)\right]^{1 / p}
$$

For $p=1$, inequality (79) is obvious. By the triangle inequality, (79), and Lemma 9, there holds

$$
\begin{aligned}
\left(\int_{\mathbb{C}^{n}}\right. & \left.\left|f_{2}(w)-\widetilde{\left(f_{2}\right)_{t}}(z)\right|^{p} \cdot\left|k_{l, z}(w)\right|^{l} e^{-l \phi(w)} d v(w)\right)^{1 / p} \\
\leq & \left(\int_{\mathbb{C}^{n}}\left|f_{2}(w)\right|^{p} \cdot\left|k_{l, z}(w)\right|^{l} e^{-l \phi(w)} d v(w)\right)^{1 / p} \\
& +\left(\int_{\mathbb{C}^{n}}\left|\widetilde{\left(f_{2}\right)_{t}}(z)\right|^{p} \cdot\left|k_{l, z}(w)\right|^{l} e^{-l \phi(w)} d v(w)\right)^{1 / p} \\
= & {\left[\left(\widetilde{\left.f_{2}\right|^{p}}\right)_{l}(z)\right]^{1 / p}+\left|\widetilde{\left(f_{2}\right)_{t}}(z)\right|^{1 / p} } \\
\leq & {\left[\left(\widetilde{\left|f_{2}\right|^{p}}\right)_{l}(z)\right]^{1 / p}+\left[\left(\widetilde{\left.f_{2}\right|^{p}}\right)_{t}(z)\right]^{1 / p} \leqslant\left\|f_{2}\right\|_{B A_{r}^{p} .} }
\end{aligned}
$$

By (78), (80), and the triangle inequality, one can get

$$
\begin{gathered}
\left(\int_{\mathbb{C}^{n}}\left|f(w)-\tilde{f}_{t}(z)\right|^{p} \cdot\left|k_{l, z}(w)\right|^{l} e^{-l \phi(w)} d v(w)\right)^{1 / p} \\
\quad \leq\left\|f_{1}\right\|_{B O_{r}}+\left\|f_{2}\right\|_{B A_{r}^{p} .} \\
(\mathrm{C}) \Rightarrow(\mathrm{A}) \text { : by (18) and }\left|B^{r}(z)\right| \simeq \rho(z)^{2 n} \text {, we get } \\
\quad \frac{1}{\left|B^{r}(z)\right|} \simeq\left|k_{l, z}(w)\right|^{l} e^{-l \phi(w)}, \quad w \in B^{r}(z) .
\end{gathered}
$$

Hence, there holds

$$
\begin{aligned}
& \frac{1}{\left|B^{r}(z)\right|} \int_{B^{r}(z)}\left|f(w)-\tilde{f}_{t}(z)\right|^{p} d v(w) \\
& \quad \int_{B^{r}(z)}\left|f(w)-\tilde{f}_{t}(z)\right|^{p} \cdot\left|k_{l, z}(w)\right|^{l} e^{-l \phi(w)} d v(w) \\
& \leq \int_{\mathbb{C}^{n}}\left|f(w)-\tilde{f}_{t}(z)\right|^{p} \cdot\left|k_{l, z}(w)\right|^{l} e^{-l \phi(w)} d v(w) .
\end{aligned}
$$

By Proposition 4 with $c(z)=\tilde{f}_{t}(z)$, we have $f \in \mathrm{BMO}_{r}^{p}$.

The estimation (63) comes from (68), (72), (81), and (83). The proof is finished.

\section{Bounded Hankel Operators}

In this section, we are devoted to characterizing complexvalued functions $f$ in $\mathcal{S}$ such that the big Hankel operators $H_{f}$ and $H_{\bar{f}}$ are simultaneously bounded from $F^{p}(\phi)$ into $L^{p}(\phi)$ with $1 \leq p<\infty$.

Theorem 12. Let $1 \leq p<\infty, 0<r<\alpha, \phi \in \mathscr{W}$, and $f \in \mathcal{S}$. If $f \in B O_{r}$, then $H_{f}: F^{p}(\phi) \longrightarrow L^{p}(\phi)$ is bounded. Moreover,

$$
\left\|H_{f}\right\| \lesssim\|f\|_{B O_{r}} .
$$

Proof. By Lemma 3 and Proposition 7, we have

$$
\begin{aligned}
\left|H_{f} g(z)\right| e^{-\phi(z)} & =|(I-P)(f g)(z)| e^{-\phi(z)} \\
& \leq e^{-\phi(z)} \int_{\mathbb{C}^{n}}|f(z)-f(w)| \cdot|g(w)| \cdot\left|K_{\phi}(z, w)\right| e^{-2 \phi(w)} d v(w) \\
& \leq\|f\|_{B O_{r}} \int_{\mathbb{C}^{n}}(d(z, w)+1) \rho(z)^{-n} \rho(w)^{-n} e^{-\sigma d(z, w)}|g(w)| e^{-\phi(w)} d v(w) .
\end{aligned}
$$

By Lemma 10, one can get

$$
\int_{\mathbb{C}^{n}} \rho(z)^{-n} \rho(w)^{-n} e^{-(1 / 2) \sigma d(z, w)} \leq C .
$$

By (75) and (86), there holds

$$
\left|H_{f} g(z)\right| e^{-\phi(z)} \lesssim\|f\|_{B O_{r}}\|g\|_{L^{\infty}(\phi)},
$$

for every $z \in \mathbb{C}^{n}$. It follows that $H_{f}$ is bounded from $F^{\infty}(\phi)$ to $L^{\infty}(\phi)$ and $\left\|H_{f}\right\| \lesssim\|f\|_{B O_{r}}$.

For $p=1$, applying Fubini's theorem, (75), and (86), we have

$$
\begin{aligned}
\int_{\mathbb{C}^{n}}\left|H_{f} g(z)\right| e^{-\phi(z)} d v(z) \leqslant & \|f\|_{B O_{r}} \int_{\mathbb{C}^{n}}|g(w)| e^{-\phi(w)} d v(w) \int_{\mathbb{C}^{n}}(d(z, w) \\
& +1) \rho(z)^{-n} \rho(w)^{-n} e^{-\sigma d(z, w)} d v(z) \\
& \leqslant\|f\|_{B O_{r}}\|g\|_{L^{1}(\phi)} .
\end{aligned}
$$

Thus, we see that $H_{f}: F^{1}(\phi) \longrightarrow L^{1}(\phi)$ is bounded and $\left\|H_{f}\right\| \lesssim\|f\|_{B O_{r}}$.

By the Riesz-Thorin interpolation theorem, we conclude that $H_{f}: F^{p}(\phi) \longrightarrow L^{p}(\phi)$ is bounded for all $1 \leq p<\infty$ and $\left\|H_{f}\right\| \lesssim\|f\|_{B O_{r}}$

Lemma 13. Let $1 \leq p<\infty, 0<r<\alpha, \phi \in \mathscr{W}$, and $f \in \mathcal{S}$. If $f$ $\in B A_{r}^{p}$, then $H_{f}$ is bounded from $F^{p}(\phi)$ to $L^{p}(\phi)$. Moreover,

$$
\left\|H_{f}\right\| \lesssim\|f\|_{B A_{r}^{p}}
$$

Proof. By Lemma 9, $f \in B A_{r}^{p}$ if and only if $|f|^{p} d v$ is a Carleson measure for $F^{P}(\phi)$. Moreover,

$$
\left(\int_{\mathbb{C}^{n}}\left|f(w) g(w) e^{-\phi(w)}\right|^{p} d v(w)\right)^{1 / p} \lesssim\|f\|_{B A_{r}^{p}}\|g\|_{L^{p}(\phi)} .
$$

for any $g \in F^{p}(\phi)$. Since $P$ is bounded on $L^{P}(\phi)$, there holds

$$
\left\|H_{f} g\right\|_{L^{p}(\phi)} \leq\|I-P\| \cdot\|f g\|_{L^{p}(\phi)} \leq\|f\|_{B A_{r}^{p}}\|g\|_{L^{p}(\phi)} .
$$

It follows that $H_{f}$ is bounded and $\left\|H_{f}\right\| \lesssim\|f\|_{B A_{r}^{p}}$

Theorem 14. Let $1 \leq p<\infty, 0<r<\alpha, \phi \in \mathscr{W}$, and $f \in \mathcal{S}$. Then, $H_{f}$ and $H_{\bar{f}}$ are both bounded from $F^{p}(\phi)$ to $L^{p}(\phi)$ if 
and only if $f \in B M O_{r}^{p}$. Moreover,

$$
\left\|H_{\bar{f}}\right\|+\left\|H_{f}\right\| \simeq\|f\|_{B M O_{r}^{p}} .
$$

Proof. By (18), for any $z \in \mathbb{C}^{n}$, we have

$$
\begin{aligned}
\left\|H_{f}\right\|^{p} & \geq\left\|H_{f} k_{p, z}\right\|_{L^{p}(\phi)}^{p}=\int_{\mathbb{C}^{n}}\left|f(w) k_{p, z}(w)-P\left(f k_{p, z}\right)(w)\right|^{p} e^{-p \phi(w)} d v(w) \\
& \geq \int_{B^{r}(z)}\left|f(w)-k_{p, z}^{-1}(w) P\left(f k_{p, z}\right)(w)\right|^{p}\left|k_{p, z}(w)\right|^{p} e^{-p \phi(w)} d v(w) \\
& \simeq \frac{1}{\left|B^{r}(z)\right|} \int_{B^{r}(z)}\left|f(w)-k_{p, z}^{-1}(w) P\left(f k_{p, z}\right)(w)\right|^{p} d v(w) .
\end{aligned}
$$

Similarly, there holds

$$
\left\|H_{\bar{f}}\right\|^{p} \gtrsim \frac{1}{\left|B^{r}(z)\right|} \int_{B^{r}(z)}\left|\bar{f}(w)-k_{p, z}^{-1}(w) \mathrm{P}\left(\bar{f} k_{p, z}\right)(w)\right|^{p} d v(w) .
$$
Thus,

By (18), for $0<r<\alpha$ and $w \in B^{r}(z)$, we have $k_{p, z}(w) \neq 0$.

$$
\begin{gathered}
k_{p, z}(w)^{-1} P\left(f k_{p, z}\right)(w) \in H\left(B^{r}(z)\right), \\
k_{p, z}(w)^{-1} P\left(\bar{f} k_{p, z}\right)(w) \in H\left(B^{r}(z)\right) .
\end{gathered}
$$

Therefore, by Lemma 5, (93), and (94), we have $f \in \mathrm{BM}$ $\mathrm{O}_{r}^{p}$. Furthermore, following the proof of Lemma 5, we will get $\|f-c(z)\|_{p, z} \leqslant\left\|H_{f}\right\|+\left\|H_{\bar{f}}\right\|$. Then, checking the proof of Proposition 4, we obtain

$$
\|f\|_{\mathrm{BMO}_{r}^{p}} \leq\left\|H_{f}\right\|+\left\|H_{\bar{f}}\right\| .
$$

Conversely, suppose $f \in \mathrm{BMO}_{r}^{p}$. By Theorem $11, f$ has a decomposition $f=f_{1}+f_{2}$ where $f_{1} \in B O_{r}$ and $f_{2} \in B A_{r}^{p}$. Then, by Lemma 12 and Lemma $13, H_{f}$ is bounded from $F^{p}(\phi)$ to $L^{p}(\phi)$. Moreover,

$$
\left\|H_{f}\right\| \leqslant\left\|f_{1}\right\|_{B O_{r}}+\left\|f_{2}\right\|_{B A_{r}^{p}} \simeq\|f\|_{\mathrm{BMO}_{r}^{p}} .
$$

Since $f \in \mathrm{BMO}_{r}^{p}$ if and only if $\bar{f} \in \mathrm{BMO}_{r}^{p}$, it follows that $H_{\bar{f}}$ is bounded from $F^{p}(\phi)$ to $L^{p}(\phi)$. Moreover,

$$
\left\|H_{\bar{f}}\right\| \lesssim\left\|f_{1}\right\|_{B O_{r}}+\left\|f_{2}\right\|_{B A_{r}^{p}} \simeq\|f\|_{\mathrm{BMO}_{r}^{p}} .
$$

Then, the norm estimation follows from these two inequalities above and (97). This completes the proof.

We immediately have the following corollaries.

Corollary 15. Let $1 \leq p<\infty, 0<r<\alpha, \phi \in \mathscr{W}$, and $f \in \mathcal{S}$. If $f$ is real-valued on $\mathbb{C}^{n}$, then $H_{f}$ is bounded from $F^{p}(\phi)$ to $L^{p}(\phi)$ if and only if $f \in B M O_{r}^{p}$.
Corollary 16. Let $1 \leq p<\infty, 0<r<\alpha, \phi \in \mathscr{W}$, and $f \in \mathcal{S}$. If $f$ is analytic on $\mathbb{C}^{n}$, then $H_{\bar{f}}$ is bounded from $F^{p}(\phi)$ to $L^{p}(\phi)$ if and only if $f \in B M O_{r}^{p}$.

\section{The Space VMO}

Suppose $r>0$. We say that $f$ belongs to $\mathrm{VMO}_{r}^{p}$ if $f \in \mathrm{BMO}_{r}^{p}$ and

$$
\lim _{|z| \longrightarrow \infty} M O_{r}^{p}(f)(z)=0
$$

As in the space BMO, we have the following two lemmas. The proof is similar to the corresponding case of BMO. One can prove them with easy modifications, so we omit details here.

Lemma 17. Let $1 \leq p<\infty, f \in L_{\text {loc }}^{p}\left(\mathbb{C}^{n}\right)$, and $r>0$. Then, $f$ $\in V M O_{r}^{p}$ if and only if there exists a complex-valued function $c(z)$ on $\mathbb{C}^{n}$ such that

$$
\lim _{|z| \rightarrow \infty} \frac{1}{\left|B^{r}(z)\right|} \int_{B^{r}(z)}|f(w)-c(z)|^{p} d v(w)=0 .
$$

Lemma 18. Let $1 \leq p<\infty, f \in L_{l o c}^{p}\left(\mathbb{C}^{n}\right)$, and $r>0$. If for any $z \in \mathbb{C}^{n}$, there exist functions $h_{1}, h_{2} \in H\left(B^{r}(z)\right)$ such that

$$
\begin{aligned}
& \lim _{|z| \rightarrow \infty} \frac{1}{\left|B^{r}(z)\right|} \int_{B^{r}(z)}\left|f(w)-h_{1}(w)\right|^{p} d v(w)=0, \\
& \lim _{|z| \longrightarrow \infty} \frac{1}{\left|B^{r}(z)\right|} \int_{B^{r}(z)}\left|\bar{f}(w)-h_{2}(w)\right|^{p} d v(w)=0,
\end{aligned}
$$

then $f \in V M O_{r}^{p}$.

Let $r>0$. We define $V O_{r}$ as the space of complex-valued functions $f$ in $B O_{r}$ satisfying

$$
\lim _{|z| \longrightarrow \infty} \omega_{r}(f)(z)=0
$$

Define $V A_{r}^{p}$ as the space of complex-valued functions $f$ in $B A_{r}^{p}$ satisfying

$$
\lim _{|z| \longrightarrow \infty}\left[\left(\widehat{|f|^{p}}\right)_{r}(z)\right]^{1 / p}=0
$$

The next result shows that $V O_{r}$ is independent of the radius $r$.

Lemma 19. Let $0<r_{1}, r_{2}<\alpha$. Then, $f \in V O_{r_{1}}$ if and only if $f \in V O_{r_{2}}$.

Proof. Without loss of generality, we may assume $r_{1}<r_{2}$. Since $\omega_{r_{1}}(f)(z) \leq \omega_{r_{2}}(f)(z)$, we have $f \in V O_{r_{1}}$ if $f \in V O_{r_{2}}$. 
Conversely, suppose $f \in V O_{r_{1}}$. For any $z \in \mathbb{C}^{n}$, since $f$ is continuous on $\mathbb{C}^{n}$, there holds

$$
\omega_{r_{2}}(f)(z)=\sup \left\{|f(z)-f(u)|: u \in B^{r_{2}^{-}}(z)\right\}
$$

Hence, there exists some $w \in B^{r_{2}^{-}}(z)$ such that

$$
\omega_{r_{2}}(f)(z)=|f(z)-f(w)|
$$

For a sufficiently small $\varepsilon>0,(1+\varepsilon) r_{2}<1$, we can apply Lemma 6 with $r=(1+\varepsilon) r_{2}$ and obtain

$$
B^{r_{2}^{-}}(z) \subseteq B^{(1+\varepsilon) r_{2}}(z) \subseteq B_{\rho}\left(z, \frac{(1+\varepsilon) r_{2}}{1-(1+\varepsilon) r_{2}}\right)
$$

Therefore,

$$
d(z, w)<\frac{(1+\varepsilon) r_{2}}{1-(1+\varepsilon) r_{2}}
$$

Let $\varepsilon \longrightarrow 0$, we get $d(z, w) \leq r_{2} /\left(1-r_{2}\right)$. We then follow a similar argument as Proposition 7 . We replace points

$$
z_{0}=z, z_{1}, \cdots, z_{N}=w
$$

on the geodesic in the $d$-metric from $z$ to $w$ in such a way: $d\left(z_{i}, z_{i-1}\right)=M_{r_{1}}$ for $i=1,2, \cdots, N-1$ and $d\left(z_{N}, z_{N-1}\right) \leq M_{r_{1}}$ where $M_{r_{1}}=r_{1} /\left(1+2 r_{1}\right)$ is an appropriate constant such that $z_{i} \in B^{r_{1}}\left(z_{i-1}\right)$. We will get a similar inequality as (50):

$N \leq\left(2+\frac{1}{r_{1}}\right)(d(z, w)+1) \leq\left(2+\frac{1}{r_{1}}\right)\left(\frac{r_{2}}{1-r_{2}}+1\right)=: N_{0}$.

Since $f \in V O_{r_{1}}$, for any $\varepsilon>0$, there exists some positive number $M_{1}$ such that

$$
\omega_{r_{1}}(f)(z)<\frac{\varepsilon}{N_{0}}, \quad|z|>M_{1} .
$$

By the triangle inequality, we get

$$
\begin{aligned}
\left|z-z_{i}\right| & \leq\left|z-z_{1}\right|+\cdots+\left|z_{i}-z_{i-1}\right| \leq r_{1} \rho(z)+\cdots+r_{1} \rho\left(z_{i-1}\right) \\
& \leq N_{0} r_{1} \sup _{z \in \mathbb{C}^{n}} \rho(z)=: M_{2} .
\end{aligned}
$$

For $|z| \geq M_{1}+M_{2}$, we clearly have $\left|z_{i}\right| \geq|z|-\left|z_{i}-z\right| \geq$ $M_{1}$. Thus, if we set $M_{0}=M_{1}+M_{2}$, then for $|z|>M_{0}$, there holds

$$
\begin{aligned}
\omega_{r_{2}}(f)(z) & =|f(z)-f(w)| \leq \sum_{i=1}^{N}\left|f\left(z_{i}\right)-f\left(z_{i-1}\right)\right| \\
& \leq \sum_{i=1}^{N} \omega_{r_{1}}(f)\left(z_{i}\right) \leq \frac{N \varepsilon}{N_{0}} \leq \varepsilon .
\end{aligned}
$$

The proof is finished.

A positive Borel measure $\mu$ on $\mathbb{C}^{n}$ is called a vanishing Carleson measure if

$$
\lim _{j \longrightarrow \infty} \int_{\mathbb{C}^{n}}\left|g_{j}(w) e^{-\phi(w)}\right|^{p} d \mu(w)=0
$$

whenever $\left\{g_{j}\right\}_{j=1}^{\infty}$ is a bounded sequence in $F^{p}(\phi)$ and converges to 0 uniformly on any compact subset of $\mathbb{C}^{n}$ as $j$ $\longrightarrow \infty$.

The following lemma characterizes the vanishing Carleson measure, and one can find it from Theorem 3.9 in [3].

Lemma 20. Let $\mu$ be a finite positive Borel measure on $\mathbb{C}^{n}$. Assume $0<p<\infty$. Then, the following conditions are all equivalent:
(A) The measure $\mu$ is a vanishing Carleson measure
(B) The function $\tilde{\mu}_{t} \longrightarrow 0$ as $|z| \longrightarrow \infty$ for $t>0$
(C) The function $\widehat{\mu}_{r} \longrightarrow 0$ as $|z| \longrightarrow \infty$ for $0<r<\alpha$

Set $d \mu=|f|^{p} d v$. Then, we have $\widehat{\mu}_{r}(z)=\left(\widehat{|f|^{p}}\right)_{r}(z)$ and $\tilde{\mu}_{t}(z)=\left(\widetilde{|f|^{p}}\right)_{t}(z)$. The following lemma comes immediately.

Lemma 21. Let $1 \leq p<\infty$ and $f \in L_{l o c}^{p}\left(\mathbb{C}^{n}\right)$. Then, the following conditions are all equivalent:

(A) The measure $d \mu=|f|^{p} d v$ is a vanishing Carleson measure

(B) The function $\left(\widetilde{|f|^{p}}\right)_{t} \longrightarrow 0$ as $|z| \longrightarrow \infty$ for $t>0$

(C) The function $\left(\widehat{|f|^{p}}\right)_{r} \longrightarrow 0$ as $|z| \longrightarrow \infty$ for $0<r<\alpha$, i.e., $f \in V A_{r}^{p}$

From the previous lemma, one can see that the space $V A_{r}^{p}$ is independent of the choice of $r \in(0, \alpha)$. So we will write $V A^{p}$ for short.

By the following decomposition theorem, we know that the space $\mathrm{VMO}_{r}^{p}$ is independent of the choice of $r \in(0, \alpha)$.

Theorem 22. Let $0<r<\alpha, 1 \leq p<\infty$, and $0<t, l<\infty$. Then, the following conditions are all equivalent:

(A) $f \in V M O_{r}^{p}$ 
(B) $f$ admits a decomposition $f=f_{1}+f_{2}$ where $f_{1} \in V O_{r}$ and $f_{2} \in V A_{r}^{p}$

(C) The function $\int_{\mathbb{C}^{n}}\left|f(w)-\tilde{f}_{t}(z)\right|^{p} \cdot\left|k_{l, z}(w)\right|^{l} e^{-l \phi} d v(w)$ $\longrightarrow 0$ as $|z| \longrightarrow \infty$

Proof. The proof is similar to Theorem 11 . Notice that $\left\{k_{l, z}\right\}$ converges uniformly to 0 as $|z| \longrightarrow \infty$ on any compact subset of $\mathbb{C}^{n}$. One can conduct the same argument to prove that (B) implies (C).

\section{Compact Hankel Operators}

Theorem 23. Let $1 \leq p<\infty, 0<r<\alpha, \phi \in \mathscr{W}$, and $f \in \mathcal{S}$. Then, $H_{f}$ and $H_{\bar{f}}$ are both compact from $F^{p}(\phi)$ to $L^{p}(\phi)$ if and only if $f \in V M O_{r}^{p}$.

Proof. Notice that $\left\{k_{p, z}\right\}$ converges to 0 on any compact subset of $\mathbb{C}^{n}$. Similar to the proof of Theorem 14, we have

$$
\begin{aligned}
& \lim _{|z| \longrightarrow \infty} \frac{1}{\left|B^{r}(z)\right|} \int_{B^{r}(z)}\left|f(w)-k_{p, z}^{-1}(w) P\left(f k_{p, z}\right)(w)\right|^{p} d v(w)=0, \\
& \lim _{|z| \longrightarrow \infty} \frac{1}{\left|B^{r}(z)\right|} \int_{B^{r}(z)}\left|\bar{f}(w)-k_{p, z}^{-1}(w) P\left(\bar{f} k_{p, z}\right)(w)\right|^{p} d v(w)=0 .
\end{aligned}
$$

By (95), (96), and Lemma 18, we get $f \in \mathrm{VMO}_{r}^{p}$.

Conversely, suppose $f \in \mathrm{VMO}_{r}^{p}$. Let $f_{R}:=f \chi_{B(0, R)}$ where $\chi_{B(0, R)}$ is the characteristic function of $B(0, R)$. Since $f \in$ $\mathrm{VMO}_{r}^{p}$, for any $\varepsilon>0$, there is some $R>0$ such that $M O_{r}^{p}(f$ )$(z)<\varepsilon$ when $|z|>R / 2$. Without loss of generality, we may suppose $r<R / 4 M$ where $M=\sup _{z \in \mathbb{C}^{n}} \rho(z)$. Then, we have $M O_{r}^{p}\left(f-f_{R}\right)(z)=M O_{r}^{p}(f)(z)<\varepsilon$ for $|z|>2 R$. Therefore, $\left\|f-f_{R}\right\|_{\mathrm{BMO}_{r}^{p}} \longrightarrow 0$ as $R \longrightarrow \infty$. Applying Theorem 14 to $f-f_{R}$, there holds

$$
\left\|H_{f-f_{R}}\right\| \lesssim\left\|f-f_{R}\right\|_{\mathrm{BMO}_{r}^{p}}
$$

By the fact that the space of compact operators is norm closed, we only need to prove that $H_{f_{R}}: F^{p}(\phi) \longrightarrow L^{p}(\phi)$ is compact.

Fix $R>0$. Let $\left\{g_{j}\right\}_{j=1}^{\infty}$ be a bounded sequence in $F^{p}(\phi)$ that converges to 0 uniformly on any compact subset of $\mathbb{C}^{n}$. From Theorem 11, we know that $\widehat{\left(f_{R}\right)}$, belongs to $B O_{r}$ , since $f_{R} \in \mathrm{BMO}_{r}^{p}$. It is clear that $\widehat{\left(f_{R}\right)_{r}}$ has compact support. For $z$ in some compact subset of $\mathbb{C}^{n}$, using Proposition 7, we obtain

$$
\left|{\widehat{\left(f_{R}\right)_{r}}}_{r}(z)-\widehat{\left(f_{R}\right)_{r}}(0)\right| \lesssim d(z, 0)+1<\infty .
$$

Hence, $\widehat{\left(f_{R}\right)}$ is bounded. From Lemma 3.3 of [3], we know that

$$
\begin{aligned}
& \int_{\mathbb{C}^{n}}\left|g_{j}(z)\right|^{p} e^{-p \phi(z)}\left|f_{R}\right|^{p}(z) d v(z) \\
& \quad \leq C \int_{\mathbb{C}^{n}}\left|g_{j}(z)\right|^{p} e^{-p \phi(z)}\left(\widehat{\left|f_{R}\right|^{p}}\right)_{r}(z) d v(z) .
\end{aligned}
$$

Therefore,

$$
\begin{aligned}
\left\|f_{R} g_{j}\right\|_{L^{p}(\phi)} & =\left(\int_{\mathbb{C}^{n}}\left|f_{R}(z) g_{j}(z)\right|^{p} e^{-p \phi(z)} d v(z)\right)^{1 / p} \\
& \leq\left(\int_{\mathbb{C}^{n}}\left|g_{j}(z)\right|^{p} e^{-p \phi(z)}\left(\widehat{\left|f_{R}\right|^{p}}\right)_{r}(z) d v(z)\right)^{1 / p} \\
& =\left(\int_{E}\left|g_{j}(z)\right|^{p} e^{-p \phi(z)} \frac{1}{\left|B^{r}(z)\right|} \int_{B^{r}(z)}\left|f_{R}(w)\right|^{p} d v(w) d v(z)\right)^{1 / p},
\end{aligned}
$$

where $E$ is the support of $\left(\mid \widehat{\left.f_{R}\right|^{p}}\right)_{r}$. We also have

$$
\begin{aligned}
& \left|f_{R}(w)-\widehat{\left(f_{R}\right)_{r}}(z)+{\widehat{\left(f_{R}\right)}}_{r}(z)\right|^{p} \\
& \quad \lesssim\left|f_{R}(w)-\widehat{\left(f_{R}\right)_{r}}(z)\right|^{p}+\left|\widehat{\left(f_{R}\right)_{r}}(z)\right|^{p} .
\end{aligned}
$$

For every $\varepsilon>0$, there is some $j_{0}$ such that

$$
\sup _{z \in E}\left|g_{j}(z)\right|<\varepsilon
$$

for $j \geq j_{0}$. Consequently,

$$
\begin{aligned}
\left\|f_{R} g_{j}\right\|_{L^{p}(\phi)} \lesssim & \left(\int _ { E } | g _ { j } ( z ) | ^ { p } e ^ { - p \phi ( z ) } \frac { 1 } { | B ^ { r } ( z ) | } \int _ { B ^ { r } ( z ) } \left[\left|f_{R}(w)-\widehat{\left(f_{R}\right)_{r}}(z)\right|^{p}\right.\right. \\
& \left.\left.+\left|\widehat{\left(f_{R}\right)_{r}}(z)\right|^{p}\right] d v(w) d v(z)\right)^{1 / p} \\
= & \left(\int _ { E } | g _ { j } ( z ) | ^ { p } e ^ { - p \phi ( z ) } \left[M O_{r}^{p}\left(f_{R}\right)(z)^{p}\right.\right. \\
& \left.\left.+\left|\widehat{\left(f_{R}\right)_{r}}(z)\right|^{p}\right] d v(z)\right)^{1 / p} \\
\lesssim & \varepsilon\left\|f_{R}\right\|_{\mathrm{BMO}_{r}^{p}}+\varepsilon \mid \widehat{\left(f_{R}\right)_{r}} \|_{L^{\infty}\left(\mathbb{C}^{n}, d v\right)}
\end{aligned}
$$

for $j \geq j_{0}$. This means that $\lim _{j \longrightarrow \infty}\left\|f_{R} g_{j}\right\|_{L^{p}(\phi)}=0$. Since $f_{R} g_{j} \in L^{p}(\phi)$ and $P$ is bounded on $L^{p}(\phi)$, there holds

$$
\left\|H_{f_{R}} g_{j}\right\|_{L^{p}(\phi)} \leq\|I-P\| \cdot\left\|f_{R} g_{j}\right\|_{L^{p}(\phi)}
$$

Thus, $H_{f_{R}}$ is compact. Since $f \in \mathrm{VMO}_{r}^{p}$ if and only if $\bar{f}$ $\in \mathrm{VMO}_{r}^{p}, H_{\bar{f}}$ is also compact.

Corollary 24. Let $1 \leq p<\infty, 0<r<\alpha, \phi \in \mathscr{W}$, and $f \in \mathcal{S}$. If $f \in V O_{r}$, then $H_{f}: F^{p}(\phi) \longrightarrow L^{p}(\phi)$ is compact. 
Corollary 25. Let $1 \leq p<\infty, 0<r<\alpha, \phi \in \mathscr{W}$, and $f \in \mathcal{S}$. If $f \in V A_{r}^{p}$, then $H_{f}: F^{p}(\phi) \longrightarrow L^{p}(\phi)$ is compact.

Corollary 26. Let $1 \leq p<\infty, 0<r<\alpha, \phi \in \mathscr{W}$, and $f \in \mathcal{S}$. If $f$ is real-valued on $\mathbb{C}^{n}$, then $H_{f}$ is compact from $F^{p}(\phi)$ to $L^{p}(\phi)$ if and only if $f \in V M O_{r}^{p}$.

Corollary 27. Let $1 \leq p<\infty, 0<r<\alpha, \phi \in \mathscr{W}$, and $f \in \mathcal{S}$. If $f$ is analytic on $\mathbb{C}^{n}$, then $H_{\bar{f}}$ is compact from $F^{p}(\phi)$ to $L^{p}(\phi)$ if and only if $f \in V M O_{r}^{p}$.

\section{Data Availability}

No data were used in this study.

\section{Conflicts of Interest}

The authors declare that they have no conflicts of interest.

\section{Acknowledgments}

This project is supported by the National Natural Science Foundation of China (Grant No. 11971125).

\section{References}

[1] G. M. Dall'Ara, "Pointwise estimates of weighted Bergman kernels in several complex variables," Advances in Mathematics, vol. 285, pp. 1706-1740, 2015.

[2] X. Lv, "Bergman projections on weighted Fock spaces in several complex variables," Journal of Inequalities and Applications, vol. 2017, no. 1, 2017.

[3] H. Arroussi and C. Tong, "Weighted composition operators between large Fock spaces in several complex variables," Journal of Functional Analysis, vol. 277, no. 10, pp. 3436-3466, 2019.

[4] Y. Liu and X. Wang, "Duality of large Fock spaces in several complex variables and compact localization operators," Journal of Function Spaces, vol. 2021, Article ID 6619459, 17 pages, 2021.

[5] K. Zhu, Analysis on Fock Spaces, Springer Verlag, New York, 2012.

[6] Z. Hu and X. Lv, "Toeplitz operators on Fock spaces $F_{(\varphi)}^{p}$," Integral Equations and Operator Theory, vol. 80, no. 1, pp. 33-59, 2014.

[7] A. P. Schuster and D. Varolin, "Toeplitz operators and Carleson measures on generalized Bargmann-Fock spaces," Integral Equations and Operator Theory, vol. 72, no. 3, pp. 363-392, 2012.

[8] K. Zhu, Operator Theory in the Function Spaces, 2nd Edition, Mathematical Surveys and Monographs 138, American Mathematical Society, 2007.

[9] W. Bauer, "Mean oscillation and Hankel operators on the Segal-Bargmann space," Integral Equations and Operator Theory, vol. 52, no. 1, pp. 1-15, 2005.

[10] Z. Hu and X. Lv, "Hankel operators on weighted Fock spaces (in Chinese)," Scientia Sinica Mathematica, vol. 46, pp. 141156, 2016.
[11] Z. H. Tu and X. F. Wang, "Mean oscillation and Hankel operators on Fock-type spaces," Acta Mathematica Sinica, vol. 37, no. 7, pp. 1089-1108, 2021.

[12] X. Wang, G. Cao, and K. Zhu, "BMO and Hankel operators on Fock-type spaces," Journal of Geometric Analysis, vol. 25, no. 3, pp. 1650-1665, 2015.

[13] Z. Hu and E. M. Wang, "Hankel operators between Fock spaces," Integral Equations and Operator Theory, vol. 90, no. 3, 2018.

[14] X. Lv, "Hankel operators on Fock spaces $F^{p}(\varphi)$," Complex Variables and Elliptic Equations, vol. 64, pp. 1-12, 2018.

[15] X. Lv and Z. Hu, "On Hankel operators between Fock spaces," Banach Journal of Mathematical Analysis, vol. 14, no. 3, pp. 871-893, 2020.

[16] J. Pau, R. Zhao, and K. Zhu, "Weighted BMO and Hankel operators between Bergman spaces," Indiana University Mathematics Journal, vol. 65, no. 5, pp. 1639-1673, 2016.

[17] Z. Hu, "Mean value properties of pluriharmonic functions," Journal of Mathematics, vol. 13, 335 pages, 1993. 